\title{
GENERIC SPLITTING FIELDS OF COMPOSITION ALGEBRAS
}

\author{
BY \\ J. C. FERRAR( ${ }^{(1)}$
}

Witt [7] proved that one can assign to each generalized quaternion algebra $\mathscr{A}$ over a field $K$, a field $F(\mathscr{A})$ containing $K$ which splits $\mathscr{A}$ and has the property: if $F(\mathscr{A})$ splits a quaternion algebra $\mathscr{B}$ over $K$ then either $\mathscr{B}$ is split over $K$ or $\mathscr{B}$ is isomorphic to $\mathscr{A}$. Amitsur [2] has generalized this result to obtain generic splitting fields for all central simple associative algebras of dimension greater than one over $K$ (cf. Roquette [6]). In this paper we generalize the result of Witt in another direction, studying splitting fields of composition algebras of dimension greater than one over $K$ of characteristic other than two. We assign to each such algebra $\mathscr{C}$, a field $F(\mathscr{C})$ containing $K$, prove that $F(\mathscr{C})$ is an invariant under isomorphisms, and prove

THEOREM 2. Let $\mathscr{C}$ be a composition algebra of dimension greater than one over $K$. Then

1. $\mathscr{C}_{F(\mathscr{C})}$ is split.

2. If $F \supseteq K$ is any field, then $\mathscr{C}_{F}$ is split if and only if there is a $K$-place of $F(\mathscr{C})$ into $F \cup \infty$.

3. If $\mathscr{C}^{\prime}$ is any composition algebra over $K$ such that $\mathscr{C}_{F(\mathscr{C})}^{\prime}$ is split, then either $\mathscr{C}^{\prime}$ is split or $\mathscr{C}$ is isomorphic to a subalgebra of $\mathscr{C}^{\prime}$.

Thus we generalize the result of Witt to quadratic and generalized Cayley algebras.

I. Composition algebras. A composition algebra $\mathscr{C}$ over a field $K$ is an algebra over $K$, with identity 1 , together with a nondegenerate quadratic form $N$ such that $N(x y)=N(x) N(y)$ for any $x, y$ in $\mathscr{C}$. The structure of such algebras has been completely determined and we refer to [1] or [4] for proofs of the following results.

1. A composition algebra $\mathscr{C}$ is alternative with involution $\tau: \alpha 1+u \rightarrow \alpha 1-u$, for $u$ orthogonal to 1 with respect to the nondegenerate, symmetric, bilinear form $N(x, y)=\frac{1}{2}\{N(x+y)-N(x)-N(y)\}$. Each $x \in \mathscr{C}$ can be uniquely represented in the form $x=\alpha 1+u, \alpha \in K, N(u, 1)=0$ and one has $N(x) 1=(\alpha 1+u)(\alpha 1-u)$.

If $V$ is a subspace of $\mathscr{C}$, we shall denote by $V^{\perp}$ the orthogonal complement of $V$ in $\mathscr{C}$ with respect to $N(x, y)$.

2. If $\mathscr{B}$ is a composition subalgebra of $\mathscr{C}$ (necessarily having associated quadratic form the restriction of $N$ to $\mathscr{B})$, and $u \in \mathscr{B}^{\perp} \subseteq C, N(u) \neq 0$, then $\mathscr{B}+\mathscr{B} u$,

Received by the editors August 28, 1966.

(1) Research partially supported by the National Science Foundation Grant GP-6368. 
$\mathscr{B} u=\{b u \mid b \in \mathscr{B}\}$, is a composition subalgebra of $\mathscr{C}$ with structure determined completely by the structure of $\mathscr{B}$ and the element $N(u) \in K . \mathscr{B} u$ is orthogonal to $\mathscr{B}$ with respect to the nondegenerate form $N(x, y)$ and hence $\operatorname{dim}(\mathscr{B}+\mathscr{B} u)=2 \operatorname{dim} \mathscr{B}$.

3. Every composition algebra $\mathscr{C}$ has dimension $1,2,4$, or 8 over $K$ and possesses composition subalgebras of dimension $2^{e}$ for all $e$ such that $2^{e} \leqq \operatorname{dim} \mathscr{C}$.

4. If $\varphi$ is an isomorphism from a composition algebra $\mathscr{C}$ with quadratic form $N$ onto a composition algebra $\mathscr{C}^{\prime}$ with quadratic form $N^{\prime}$, then $N^{\prime}(x \varphi)=N(x)$ for all $x \in \mathscr{C}$.

5. A composition algebra is called split if there is $u \in \mathscr{C}, u \neq 0$, such that $N(u)=0$. If $\mathscr{C}$ is split, the form $N(x, y)$ has maximal Witt index. If $\mathscr{C}$ is not split, $\mathscr{C}$ is a division algebra.

6. If $F \supseteq K$ is a field, the algebra $\mathscr{C}_{F}=\mathscr{C} \otimes_{K} F$ is again a composition algebra (over $F$ ) with associated quadratic form $N_{F}$, the natural extension of $N$ to $\mathscr{C}_{F}$.

For convenience we shall denote by $\lambda x, \lambda \in F, x \in \mathscr{C}$, the element $\lambda \otimes x$ of $\mathscr{C}_{F}$.

II. Construction of the generic splitting field. We assume now that $\mathscr{C}$ is an arbitrary composition algebra of dimension $2^{k}, k>0$, over $K$ of characteristic other than two. Let $u_{i}, 1 \leqq i \leqq m+1, m=2^{k-1}$, be elements of $\mathscr{C}$ such that $N\left(u_{i}\right) \neq 0$ for all $i, N\left(u_{i}, u_{j}\right)=0$ for $i \neq j$, and $u_{i}, 1 \leqq i \leqq m$, span a composition algebra $\mathscr{B} \subseteq \mathscr{C}$. We take $L(\mathscr{C})$ to be the rational function field in $m-1$ indeterminates $x_{2}, \ldots, x_{m}$ over $K$, assuming as a convention that this will be $K$ if $m=1$, and define

$$
\lambda(u)=N\left(u_{1}\right)^{-1} N\left(\sum_{2}^{m} u_{i} x_{i}+u_{m+1}\right)=N\left(u_{1}\right)^{-1}\left(\sum_{2}^{m} x_{i}^{2} N\left(u_{i}\right)+N\left(u_{m+1}\right)\right)
$$

in $L(\mathscr{C})$.

The generic splitting field $F(\mathscr{C})$ is defined as follows: $F(\mathscr{C})=L(\mathscr{C})$ if $\mathscr{C}$ is split; $F(\mathscr{C})=L(\mathscr{C})\left((-\lambda(u))^{1 / 2}\right)$ if $\mathscr{C}$ is not split.

We show now that $F(\mathscr{C})$ is dependent, up to isomorphism, only on $\mathscr{C}$, and not on the choice of the $u_{i}$, proving first

LeMMA 1. Let $\mathscr{C}$ be a composition division algebra over $K, u_{i}, v_{i}, 1 \leqq i \leqq m+1$ sets of elements of $\mathscr{C}$ satisfying the conditions above and such that $u_{i}, 1 \leqq i \leqq m$, and $v_{i}$, $1 \leqq i \leqq m$, span the same subalgebra $\mathscr{B}$ of $\mathscr{C}$. Then $L(\mathscr{C})\left((-\lambda(u))^{1 / 2}\right)$ is isomorphic to $L(\mathscr{C})\left((-\lambda(v))^{1 / 2}\right)$.

Proof. By (2), $\mathscr{C}=\mathscr{B}+\mathscr{B} u_{m+1}$ and $\mathscr{B}^{\perp}=\mathscr{B} u_{m+1}$. Thus there is $b \in \mathscr{B}$ such that $v_{m+1}=b u_{m+1}, N(b) \neq 0$. Since $b u_{i}, 1 \leqq i \leqq m$ span $\mathscr{B}$,

$$
\alpha v_{1}+\sum_{2}^{m} x_{i} v_{i}+v_{m+1}=\sum_{1}^{m} \xi_{i}\left(b u_{i}\right)+b u_{m+1}=b\left(\sum_{1}^{m} \xi_{i} u_{i}+u_{m+1}\right)
$$

for any $\alpha \in L(\mathscr{C})\left((-\lambda(v))^{1 / 2}\right)$, where $\xi_{i}, 1 \leqq i \leqq m$, are $K$-linear combinations of $\alpha$ and the $x_{i}, 2 \leqq i \leqq m$, and conversely. For $\alpha=(-\lambda(v))^{1 / 2}$,

$$
0=N\left(\alpha v_{1}+\sum_{2}^{m} x_{i} v_{i}+v_{m+1}\right)=N(b) N\left(\sum_{1}^{m} \xi_{i} u_{i}+u_{m+1}\right)
$$


and, since $N(b) \neq 0, \xi_{1}^{2}=-N\left(u_{1}\right)^{-1}\left(\sum_{2}^{m} \xi_{i}^{2} N\left(u_{i}\right)+N\left(u_{m+1}\right)\right)$. Since the $\xi_{i}$ generate $L(\mathscr{C})\left((-\lambda(v))^{1 / 2}\right)$ over $K$, it follows that there is an isomorphism of $L(\mathscr{C})\left((-\lambda(u))^{1 / 2}\right)$ onto $L(\mathscr{C})\left((-\lambda(v))^{1 / 2}\right)$ mapping $x_{i}$ onto $\xi_{i}, 2 \leqq i \leqq m$, and $(-\lambda(u))^{1 / 2}$ onto $\xi_{1}$.

We shall obtain our results on the independence of $F(\mathscr{C})$ from the choice of the $u_{i}$, and on the invariance of $F(\mathscr{C})$ under isomorphism of $\mathscr{C}$, as corollaries to

THEOREM 1. Let $u_{i}, v_{i}, 1 \leqq i \leqq m+1$ be elements of a division composition algebra $\mathscr{C}$, satisfying the criteria given for the $u_{i}$ in defining $F(\mathscr{C})$. Let $u_{i}, 1 \leqq i \leqq m$, span the subalgebra $\mathscr{B}$ and let $v_{i}, 1 \leqq i \leqq m$, span the subalgebra $\mathscr{B}^{\prime}$. Then $L(\mathscr{C})\left((-\lambda(u))^{1 / 2}\right)$ is isomorphic to $L(\mathscr{C})\left((-\lambda(v))^{1 / 2}\right)$.

Proof. We consider the separate cases $m=1,2$, or 4 .

Case 1. $m=1$. The only one-dimensional composition subalgebra of $\mathscr{C}$ is $K 1$, hence $\mathscr{B}=\mathscr{B}^{\prime}$ and the result follows from Lemma 1 .

Case 2. $m=2$. If $\mathscr{B}=\mathscr{B}$ ', Lemma 1 again yields the desired result. Thus we may assume $\mathscr{B} \cap \mathscr{B}^{\prime}=K 1$.

If $1, u$ are an orthogonal basis for $\mathscr{B}, v \in \mathscr{B}^{\perp}$, then $1, v$ also span a subalgebra, say $\mathscr{D}$, of $\mathscr{C}$. Taking $u_{1}=1, u_{2}=u, u_{3}=v, u_{i}^{\prime}=1, u_{2}^{\prime}=v, u_{3}^{\prime}=u$, we see easily that since $\lambda(u)=N(u) x_{1}^{2}+N(v), \lambda\left(u^{\prime}\right)=N(v) x_{1}^{2}+N(u)$, the mapping taking $x_{1}$ onto $x_{1}^{-1}$, $(-\lambda(u))^{1 / 2}$ onto $x_{1}^{-1}\left(-\lambda\left(u^{\prime}\right)\right)^{1 / 2}$ determines an isomorphism of $L(\mathscr{C})\left((-\lambda(u))^{1 / 2}\right)$ onto $L(\mathscr{C})\left(\left(-\lambda\left(u^{\prime}\right)\right)^{1 / 2}\right)$.

Since $\mathscr{B}^{\perp},\left(\mathscr{B}^{\prime}\right)^{\perp}$ are two-dimensional subspaces of the three dimensional space $(K 1)^{\perp}$, there is $z \in \mathscr{B}^{\perp} \cap\left(\mathscr{B}^{\prime}\right)^{\perp}, z \neq 0$. By the above observation and Lemma 1 , $L(\mathscr{C})\left((-\lambda(u))^{1 / 2}\right), L(\mathscr{C})\left((-\lambda(v))^{1 / 2}\right)$ are isomorphic to fields $L(\mathscr{C})\left(\left(-\lambda\left(u^{\prime}\right)\right)^{1 / 2}\right)$, $L(\mathscr{C})\left(\left(-\lambda\left(v^{\prime}\right)\right)^{1 / 2}\right)$ respectively, where $u_{1}^{\prime}=1=v_{1}^{\prime}, u_{2}^{\prime}=z=v_{2}^{\prime}$. By Lemma 1 the latter fields are isomorphic and the result follows.

Case 3. $m=4$. Again, if $\mathscr{B}=\mathscr{B}{ }^{\prime}$ we are finished. To complete the proof we shall show the result follows in the event $\operatorname{dim}\left(\mathscr{B} \cap \mathscr{B}^{\prime}\right)=2$, and shall give a method of reducing the case $\mathscr{B} \cap \mathscr{B}^{\prime}=K 1$ to the case $\operatorname{dim}\left(\mathscr{B} \cap \mathscr{B}^{\prime}\right)=2$.

We show first that if $\mathscr{D}$ is a composition subalgebra of $\mathscr{B}$ of dimension 2 with orthogonal basis $1, a_{1}, a_{2} \in \mathscr{B} \cap \mathscr{D}^{\perp}, a_{3} \in \mathscr{B}^{\perp}$, and we take $u_{1}=1, u_{2}=a_{1}, u_{3}=a_{2}$, $u_{4}=a_{1} a_{2}, u_{5}=a_{3}, u_{1}^{\prime}=1, u_{2}^{\prime}=a_{1}, u_{3}^{\prime}=a_{3}, u_{4}^{\prime}=a_{1} a_{3}, u_{5}^{\prime}=a_{2}$ (such sets are easily seen to satisfy the necessary criteria for use in defining $F(\mathscr{C})$, then $L(\mathscr{C})\left((-\lambda(u))^{1 / 2}\right)$ is isomorphic to $L(\mathscr{C})\left(\left(-\lambda\left(u^{\prime}\right)\right)^{1 / 2}\right)$. For $\alpha \in L(\mathscr{C})\left((-\lambda(u))^{1 / 2}\right)$,

$$
\alpha 1+x_{1} a_{1}+x_{2} a_{2}+x_{3} a_{1} a_{2}+a_{3}=\left(\alpha 1+x_{1} a_{1}+a_{3}\right)+\left(x_{2} 1+x_{3} a_{1}\right) a_{2}
$$

and since, for $\alpha=(-\lambda(u))^{1 / 2}, \quad N\left(\alpha 1+x_{1} a_{1}+x_{2} a_{2}+x_{3} a_{1} a_{2}+a_{3}\right)=0$, we have $N\left(\left(x_{2} 1+x_{3} a_{1}\right)^{-1}\left(\alpha 1+x_{1} a_{1}+a_{3}\right)+a_{2}\right)=0$. Since $\left(x_{2} 1+x_{3} a_{1}\right)^{-1}=\left(x_{2}^{2}+x_{3}^{2} N\left(a_{1}\right)\right)^{-1}$ $\times\left(x_{2} 1-x_{3} a_{1}\right)$ by (1) we have, carrying out the multiplication term by term, and converting,

$$
N\left(\sum_{1}^{4} \xi_{i} u_{i}^{\prime}+u_{5}^{\prime}\right)=\sum_{1}^{4} \xi_{i}^{2} N\left(u_{i}^{\prime}\right)+N\left(u_{5}^{\prime}\right)=0
$$


where

$$
\begin{aligned}
& \xi_{1}=\left(x_{2}^{2}+x_{3}^{2} N\left(a_{1}\right)\right)^{-1}\left(\alpha x_{2}+x_{1} x_{3} N\left(a_{1}\right)\right) \\
& \xi_{2}=\left(x_{2}^{2}+x_{3}^{2} N\left(a_{1}\right)\right)^{-1}\left(x_{1} x_{2}-\alpha x_{3}\right) \\
& \xi_{3}=\left(x_{2}^{2}+x_{3}^{2} N\left(a_{1}\right)\right)^{-1} x_{2} \\
& \xi_{4}=-\left(x_{2}^{2}+x_{3}^{2} N\left(a_{1}\right)\right)^{-1} x_{3} .
\end{aligned}
$$

In $K\left(\xi_{1}, \xi_{2}, \xi_{3}, \xi_{4}\right) \subseteq L(\mathscr{C})\left((-\lambda(u))^{1 / 2}\right)$ are the elements

$$
\xi_{3}^{2}+\xi_{4}^{2} N\left(a_{1}\right)=\left(x_{2}^{2}+x_{3}^{2} N\left(a_{1}\right)\right)^{-1},
$$

and hence $x_{2}, x_{3} ; x_{2}\left(\alpha x_{2}+x_{1} x_{3} N\left(a_{1}\right)\right)-x_{3} N\left(a_{1}\right)\left(x_{1} x_{2}-\alpha x_{3}\right)=\alpha\left(x_{2}^{2}+x_{3}^{2} N\left(a_{1}\right)\right)$, hence $\alpha$; and finally $x_{1}$. Thus $K\left(\xi_{1}, \xi_{2}, \xi_{3}, \xi_{4}\right)=L(\mathscr{C})\left((-\lambda(u))^{1 / 2}\right)$ when $\alpha=(-\lambda(u))^{1 / 2}$, and the mapping taking $x_{i}$ onto $\xi_{i}, 2 \leqq i \leqq 4$, and $\left(-\lambda\left(u^{\prime}\right)\right)^{1 / 2}$ onto $\xi_{1}$ determines an isomorphism of $L(\mathscr{C})\left(\left(-\lambda\left(u^{\prime}\right)\right)^{1 / 2}\right)$ onto $L(\mathscr{C})\left((-\lambda(u))^{1 / 2}\right)$ since

$$
\xi_{1}^{2}=-N\left(u_{1}^{\prime}\right)^{-1}\left(\sum_{2}^{4} \xi_{i}^{2} N\left(u_{i}^{\prime}\right)+N\left(u_{5}^{\prime}\right)\right) .
$$

Now if $\mathscr{B} \cap \mathscr{B}^{\prime}=\mathscr{D}$ is two-dimensional, and $z \in \mathscr{B}^{\perp} \cap\left(\mathscr{B}^{\prime}\right)^{\perp}$, the latter intersection being nontrivial from dimensionality arguments as in Case 2 , we may use the above result and Lemma 1 to show $L(\mathscr{C})\left((-\lambda(u))^{1 / 2}\right), L(\mathscr{C})\left((-\lambda(v))^{1 / 2}\right)$ are isomorphic rispectively to fields $L(\mathscr{C})\left(\left(-\lambda\left(u^{\prime}\right)\right)^{1 / 2}\right), L(\mathscr{C})\left(\left(-\lambda\left(v^{\prime}\right)\right)^{1 / 2}\right)$ where $u_{i}^{\prime}$, $1 \leqq i \leqq 4$, and $v_{i,}^{\prime} 1 \leqq i \leqq 4$, span the same subalgebra $\mathscr{D}+\mathscr{D} z$. Lemma 1 then completes the argument.

If $\mathscr{B} \cap \mathscr{B}^{\prime}=K 1$, we have again a nontrivial $z \in \mathscr{B}^{\perp} \cap\left(\mathscr{B}^{\prime}\right)^{\perp}$ and we take subalgebras $\mathscr{D}, \mathscr{D}^{\prime}$ of dimension 2 in $\mathscr{B}, \mathscr{B}^{\prime}$ respectively. Again it follows that $L(\mathscr{C})\left((-\lambda(u))^{1 / 2}\right)$ is isomorphic to $L(\mathscr{C})\left(\left(-\lambda\left(u^{\prime}\right)\right)^{1 / 2}\right)$ where $u_{i}^{\prime}, 1 \leqq i \leqq 4$, span $\mathscr{D}+\mathscr{D} z$, and that $L(\mathscr{C})\left((-\lambda(v))^{1 / 2}\right)$ is isomorphic to $L(\mathscr{C})\left(\left(-\lambda\left(v^{\prime}\right)\right)^{1 / 2}\right)$, where $v_{i}^{\prime}, 1 \leqq i \leqq 4$, span $\mathscr{D}^{\prime}+\mathscr{D}^{\prime} z$. Since $(\mathscr{D}+\mathscr{D} z) \cap\left(\mathscr{D}^{\prime}+\mathscr{D}^{\prime} z\right)$ is the algebra spanned by 1 and $z$, we have reduced the argument to the case $\mathscr{B} \cap \mathscr{B}^{\prime}$ two-dimensional and are finished.

COROLlaRY 1. The field $F(\mathscr{C})$ is independent of the choice of the $u_{i} \in \mathscr{C}$ used in defining it.

Proof. If $\mathscr{C}$ is split, $F(\mathscr{C})$ depends only on the dimension of $\mathscr{C}$ for its definition. If $\mathscr{C}$ is not split, Theorem 1 shows the independence from $u_{i}$.

Corollary 2. If $\mathscr{C}$ is isomorphic to $\mathscr{C}^{\prime}$ then $F(\mathscr{C})$ is isomorphic to $F\left(\mathscr{C}^{\prime}\right)$.

Proof. If $\varphi$ is an isomorphism of $\mathscr{C}$ onto $\mathscr{C}^{\prime}, N^{\prime}(x \varphi)=N(x)$ for all $x \in \mathscr{C}$ by (4). If $u_{i}, 1 \leqq i \leqq m+1$, are chosen as above to define $F(\mathscr{C})$ and $u_{i}, 1 \leqq i \leqq m$, span $\mathscr{B} \subseteq \mathscr{C}$, the elements $u_{i} \varphi$ in $\mathscr{C}^{\prime}$ are orthogonal, have $N^{\prime}\left(u_{i} \varphi\right) \neq 0$ and $u_{i} \varphi, 1 \leqq i \leqq 4$, span the 
composition subalgebra $\mathscr{B} \varphi \subseteq \mathscr{C}^{\prime}$. Thus $u_{i} \varphi, 1 \leqq i \leqq m+1$ may be used to define $F\left(\mathscr{C}^{\prime}\right)$. Now $L(\mathscr{C})$ is clearly isomorphic to $L\left(\mathscr{C}^{\prime}\right)$ and

$$
\begin{aligned}
\lambda(u) & =N\left(u_{1}\right)^{-1}\left(\sum_{2}^{m} N\left(u_{i}\right) x_{i}^{2}+N\left(u_{m+1}\right)\right) \\
& =N^{\prime}\left(u_{1} \varphi\right)^{-1}\left(\sum_{2}^{m} N^{\prime}\left(u_{i} \varphi\right) x_{i}^{2}+N^{\prime}\left(u_{m+1} \varphi\right)\right)=\lambda(u \varphi)
\end{aligned}
$$

so $F(\mathscr{C})=L(\mathscr{C})\left((-\lambda(u))^{1 / 2}\right)$ is isomorphic to $L\left(\mathscr{C}^{\prime}\right)\left((-\lambda(u \varphi))^{1 / 2}\right)=F\left(\mathscr{C}^{\prime}\right)$.

III. Properties of $F(\mathscr{C})$. In this section we prove a sequence of lemmas leading to the proof of our main theorem. We first prove

Lemma 2. Let $K\left(x_{1}, \ldots, x_{n}\right)$ be the rational function field in $n$ indeterminates $x_{1}, \ldots, x_{n}, F$ a field extension of $K, \alpha_{1}, \ldots, \alpha_{n} \in F$. Then there is a $K$-place of $K\left(x_{1}, \ldots, x_{n}\right)$ into $F \cup \infty$ mapping $x_{i}$ onto $\alpha_{i}, 1 \leqq i \leqq n$.

Proof. By induction on $n$. The result is well known if $n=1$ and the place can, in fact, be defined explicitly. If $n>1$, we use the induction hypothesis, with $K$ replaced by $K\left(x_{1}\right)$ to claim there is a $K\left(x_{1}\right)$-place $\psi$ of $K\left(x_{1}\right)\left(x_{2}, \ldots, x_{n}\right)$ into $K\left(x_{1}\right)\left(\alpha_{2}, \ldots, \alpha_{n}\right)$ such that $x_{i}$ maps to $\alpha_{i}, i>1$. Now by the validity of the result for one indeterminate, there is a place $\varphi$ of $K\left(x_{1}\right)\left(\alpha_{2}, \ldots, \alpha_{n}\right)=K\left(\alpha_{2}, \ldots, \alpha_{n}\right)\left(x_{1}\right)$ into $F \cup \infty$ fixing the elements of $K\left(\alpha_{2}, \ldots, \alpha_{n}\right) \subseteq F$ and mapping $x_{1}$ onto $\alpha_{1} . \psi \varphi$ is then a $K$-place of $K\left(x_{1}, \ldots, x_{n}\right)$ into $F \cup \infty$ with the desired property.

Corollary. Let $\lambda \in K\left(x_{1}, \ldots, x_{n}\right)$ such that $K\left(x_{1}, \ldots, x_{n}\right)\left(\lambda^{1 / 2}\right)$ is a quadratic extension of $K\left(x_{1}, \ldots, x_{n}\right), \alpha_{1}, \ldots, \alpha_{n} \in F, F$ a field extension of $K$. Then there is a $K$-place $\varphi$ of $K\left(x_{1}, \ldots, x_{n}\right)$ into $F \cup \infty$ mapping $x_{i}$ onto $\alpha_{i}$ for all $1 \leqq i \leqq n$ and, if $\lambda \varphi$ is a square in $F, \varphi$ can be extended to a $K$-place of $K\left(x_{1}, \ldots, x_{n}\right)\left(\lambda^{1 / 2}\right)$ into $F \cup \infty$ mapping $\lambda$ onto a square root of $\lambda \varphi$ in $F$.

Proof. That $\varphi$ exists follows from Lemma 2. It is known (e.g., [3]), that a place from $K\left(x_{1}, \ldots, x_{n}\right)$ into $F \cup \infty$ can be extended to a place $\varphi^{\prime}$ of $K\left(x_{1}, \ldots, x_{n}\right)\left(\lambda^{1 / 2}\right)$ into $F^{\prime} \cup \infty, F^{\prime}$ the algebraic closure of $F$. Since, however, $\left(\lambda^{1 / 2}\right) \varphi^{\prime}$ must be a square root of $\lambda \varphi$ in $F^{\prime}$, and since the square roots of $\lambda \varphi$ in $F^{\prime}$ are in fact, in $F,\left(\lambda^{1 / 2}\right) \varphi^{\prime} \in F$ and $\varphi^{\prime}$ maps $K\left(x_{1}, \ldots, x_{n}\right)\left(\lambda^{1 / 2}\right)$ into $F \cup \infty$.

If $\mathscr{C}$ is a composition algebra over $K, F$ a field extension of $K$, we say $F$ splits $\mathscr{C}$ ( $F$ is a splitting field of $\mathscr{C}$ ) if $\mathscr{C}_{F}$ is split.

Lemma 3. $L=K\left(x_{1}, \ldots, x_{n}\right)$, the field of rational functions in $n$ indeterminates, $n \geqq 0$, splits $\mathscr{C}$ if and only if $\mathscr{C}$ is split over $K$.

Proof. We show that, if $K\left(x_{1}, \ldots, x_{n}\right)$ splits $\mathscr{C}, n \geqq 1$, then $K\left(x_{1}, \ldots, x_{n-1}\right)$ also splits $\mathscr{C}$ and hence, by induction, $K$ splits $\mathscr{C}$ so $\mathscr{C}$ is split.

Let $u_{1}, \ldots, u_{e}$ be an orthogonal basis for $\mathscr{C}$ with respect to $N(x, y)$. This is also an orthogonal basis for $\mathscr{C}_{L}$ over $L$ and, if $\mathscr{C}_{L}$ is split, there are $\xi_{i} \in L, 1 \leqq i \leqq e$, such 
that $N\left(\sum_{1}^{e} \xi_{i} u_{i}\right)=0$. Clearing the denominators of the $\xi_{i}$ we have, since $N(\alpha x)$ $=\alpha^{2} N(x)$ for $\alpha \in L$, polynomials $p_{i}$ in $K\left[x_{1}, \ldots, x_{n}\right]$, not all $p_{i} \equiv 0$, such that $N\left(\sum_{1}^{e} p_{i} u_{i}\right)=\sum_{1}^{e} p_{i}^{2} N\left(u_{i}\right)=0$. We assume, without loss of generality, that $x_{n}$ occurs in some $p_{i}$ and we let $k$ be the maximum of the degrees of the polynomials $p_{i}$, considered as polynomials in $x_{n}$ over $K\left(x_{1}, \ldots, x_{n-1}\right)$. We can write each $p_{i}=x_{n}^{k} q_{i}+r_{i}$ where $q_{i} \in K\left[x_{1}, \ldots, x_{n-1}\right], r_{i} \in K\left[x_{1}, \ldots, x_{n}\right], r_{i}$ of degree less than $k$ in $x_{n}$. Then $\sum_{1}^{e}\left(x_{n}^{k} q_{i}+r_{i}\right)^{2} N\left(u_{i}\right)=0$ and, since the $x_{i}$ are algebraically independent, we must have $\left(\sum_{1}^{e} q_{i}^{2} N\left(u_{i}\right)\right) x_{n}^{2 k}=0$, hence $\sum_{1}^{e} q_{i}^{2} N\left(u_{i}\right)=0$ in $K\left(x_{1}, \ldots, x_{n-1}\right)$. Thus $K\left(x_{1}, \ldots, x_{n-1}\right)$ splits $\mathscr{C}$. Induction completes the proof that $\mathscr{C}$ is split over $K$.

Conversely, if $\mathscr{C}$ is split over $K$ and $F$ is any field containing $K$, there is $u \in \mathscr{C}$, $u \neq 0$ such that $N(u)=0$. But $u \in \mathscr{C}$ implies $u \in \mathscr{C}_{F}$ so, since $N_{F}(u)=N(u)=0, \mathscr{C}_{F}$ is split. In particular $\mathscr{C}_{L}$ is split.

LemMa 4. Let $\mathscr{C}$ be a composition algebra over $K, F, F^{\prime}$ field extensions of $K, \varphi$ a $K$-place of $F$ into $F^{\prime} \cup \infty$. If $C_{F}$ is split, so is $\mathscr{C}_{F^{\prime}}$.

Proof. We show first that if $\lambda_{1}, \ldots, \lambda_{n}$ are elements of $F$, not all zero, there is some $j$ such that $\left(\lambda_{j}^{-1} \lambda_{i}\right) \varphi \in F^{\prime}, i=1, \ldots, n$. Let $j$ be such that $\lambda_{j} \neq 0$ and such that the number $t$ of $i$ for which $\left(\lambda_{j}^{-1} \lambda_{i}\right) \varphi=\infty$ is minimal. If $t=0$ we are done. If not, we may assume, without loss of generality, that $\left(\lambda_{j}^{-1} \lambda_{i}\right) \varphi=\infty, 1 \leqq i \leqq t,\left(\lambda_{j}^{-1} \lambda_{i}\right) \varphi \in F^{\prime}$, $t<i \leqq n . \quad \lambda_{t} \neq 0$ since otherwise $\left(\lambda_{j}^{-1} \lambda_{t}\right) \varphi=0 \varphi=0 \neq \infty$. Thus $\left(\lambda_{t}^{-1} \lambda_{i}\right) \varphi=\left(\left(\lambda_{j}^{-1} \lambda_{t}\right)^{-1}\right.$ $\left.\times\left(\lambda_{j}^{-1} \lambda_{i}\right)\right) \varphi=0$ for $t<i \leqq n$, and $\left(\lambda_{t}^{-1} \lambda_{t}\right) \varphi=1 \varphi=1 \in F^{\prime}$ and hence for $\lambda_{t}$ there are at most $(t-1) i$ such that $\left(\lambda_{t}^{-1} \lambda_{i}\right) \varphi=\infty$, a contradiction to the minimality of $t$. Thus $t=0$.

Now if $\mathscr{C}_{F}$ is split, and $u_{i}, i=1, \ldots, n$, are an orthogonal basis of $\mathscr{C}$ over $K$, hence of $\mathscr{C}_{F}$ over $F$ and of $\mathscr{C}_{F}$, over $F^{\prime}$, there are $\lambda_{i} \in F$ such that not all $\lambda_{i}$ are zero and $N_{F}\left(\sum_{1}^{n} \lambda_{i} u_{i}\right)=\sum_{1}^{n} \lambda_{i}^{2} N\left(u_{i}\right)=0$. For $\lambda_{j}$ such that $\left(\lambda_{j}^{-1} \lambda_{i}\right) \varphi \in F^{\prime}$ for all $i$,

$$
\sum_{1}^{n}\left(\lambda_{j}^{-1} \lambda_{i}\right)^{2} N\left(u_{i}\right)=0
$$

and hence, $\sum_{1}^{n}\left(\lambda_{j}^{-1} \lambda_{i}\right)^{2} \varphi N\left(u_{i}\right)=0$. Since $\left(\lambda_{j}^{-1} \lambda_{i}\right)^{2} \varphi=\left(\left(\lambda_{j}^{-1} \lambda_{i}\right) \varphi\right)^{2}$, it follows that $N_{F^{\prime}}\left(\sum_{1}^{n}\left(\lambda_{j}^{-1} \lambda_{i}\right) \varphi u_{i}\right)=0$ and, since $\left(\lambda_{j}^{-1} \lambda_{j}\right) \varphi=1 \neq 0, \mathscr{C}_{F^{\prime}}$ is split.

LEMMA 5. Let $\mathscr{C}$ be a division composition algebra over $K, \lambda \in K$, and suppose $L=K\left(\lambda^{1 / 2}\right)$ is a quadratic extension of $K$. Then $\mathscr{C}_{L}$ is split if and only if there is $u \in(K 1)^{\perp} \subseteq \mathscr{C}$ such that $N(u)=-\lambda$.

Proof. If there is $u \in(K 1)^{\perp}$ with $N(u)=-\lambda$, then $x=\left(\lambda^{1 / 2}\right) 1+u \in \mathscr{C}_{L}$ clearly has $N_{L}(x)=0$, so $\mathscr{C}_{L}$ is split. Conversely, if $\mathscr{C}_{L}$ is split, then there is $x=a+\left(\lambda^{1 / 2}\right) b$, $a, b \in \mathscr{C}$, such that $x \neq 0, N_{L}(x)=0$. But $N_{L}(x)=N(a)+\lambda N(b)+2 N(a, b)\left(\lambda^{1 / 2}\right)$ and thus $N(a, b)=0, N\left(a b^{-1}\right)=N(a) N(b)^{-1}=-\lambda$. Since $N\left(a b^{-1}, 1\right)=N(a, b) N\left(b^{-1}\right)=0$, $u=a b^{-1}$ satisfies the criteria.

Finally we give a slight generalization of a result of Jacobson [4], first defining subspaces $V, V^{\prime}$ of composition algebras $\mathscr{C}, \mathscr{C}^{\prime}$ respectively, to be equivalent if there 
is a nonsingular linear transformation $\varphi$ of $V$ onto $V^{\prime}$ such that $N^{\prime}(x \varphi)=N(x)$ for all $x \in V, N, N^{\prime}$ denoting the respective quadratic forms of $\mathscr{C}$ and $\mathscr{C}^{\prime}$.

LEMMA 6. If a composition algebra $\mathscr{C}$ is equitalent to a subspace of a composition algebra $\mathscr{C}^{\prime}$, then $\mathscr{C}$ is isomorphic to a subalgebra of $\mathscr{C}^{\prime}$.

Proof. The proof is essentially that of Jacobson [4]. Let $\varphi$ be the mapping of $\mathscr{C}$ into $\mathscr{C}^{\prime}$ such that $N^{\prime}(x \varphi)=N(x)$ for all $x \in \mathscr{C}$ and suppose that $\mathscr{B}, \mathscr{B}$ ' are isomorphic composition subalgebras of $\mathscr{C}, \mathscr{C}^{\prime}$ respectively. By (4), $\mathscr{B}$ and $\mathscr{B}^{\prime}$ are equivalent and, since $\mathscr{B}$ and $\mathscr{B} \varphi$ are clearly equivalent, $\mathscr{B}$ ' and $\mathscr{B} \varphi$ are equivalent subspaces of $\mathscr{C}^{\prime}$. By Witt's Theorem for bilinear forms, $\left(\mathscr{B}^{\prime}\right)^{\perp}$ and $(\mathscr{B} \varphi)^{\perp}$ are equivalent in $\mathscr{C}^{\prime}$. Thus, if there is $u \in \mathscr{B}^{\perp}$ with $N(u) \neq 0$, which is the case unless $\mathscr{B}=\mathscr{C}$, then there is $u^{\prime} \in\left(\mathscr{B}^{\prime}\right)^{\perp}$ such that $N^{\prime}\left(u^{\prime}\right)=N^{\prime}(u \varphi)=N(u)$. Then the algebras $\mathscr{B}+\mathscr{B} u, \mathscr{B}^{\prime}+\mathscr{B}^{\prime} u^{\prime}$ are composition subalgebras of $\mathscr{C}, \mathscr{C}^{\prime}$ respectively which are isomorphic by (2). Beginning with $\mathscr{B}=K 1, \mathscr{B}^{\prime}=K 1^{\prime}$ one can, in successive steps, thus construct an isomorphism of $\mathscr{C}$ into $\mathscr{C}^{\prime}$.

Since in this proof, whenever $2 \operatorname{dim} \mathscr{B}=\operatorname{dim} \mathscr{C}$, we need only produce elements $u, u^{\prime}$ in $\mathscr{B}^{\perp},\left(\mathscr{B}^{\prime}\right)^{\perp}$ respectively with $N(u)=N^{\prime}\left(u^{\prime}\right) \neq 0$, we can clearly weaken the hypotheses to obtain the

COROLlaRY. Let $\mathscr{C}$ be a 2 -dimensional composition algebra, $V$ a nonisotropic $(N(x, y)$ nondegenerate when restricted to $V)$ subspace of $\mathscr{C}$ of dimension $n+1$ which contains an $n$-dimensional composition subalgebra $\mathscr{B}$ of $\mathscr{C}$. Then if $V$ is equivalent to a subspace of a compasition algebra $\mathscr{C}^{\prime}, \mathscr{C}$ is isomorphic to a subalgebra of $\mathscr{C}^{\prime}$.

We are now prepared to restate and prove

THEOREM 2. Let $\mathscr{C}$ be a composition algebra of dimension greater than one over $K$. Then

1. $\mathscr{C}_{F(\mathscr{C})}$ is split.

2. If $F \supseteq K$ is any field, then $\mathscr{C}_{F}$ is split if and only if there is a $K$-place of $F(\mathscr{C})$ into $F \cup \infty$.

3. If $\mathscr{C}^{\prime}$ is any composition algebra over $K$ such that $\mathscr{C}_{F(\mathscr{C})}^{\prime}$ is split, then either $\mathscr{C}^{\prime}$ is split over $K$ or $\mathscr{C}$ is isomorphic to a subalgebra of $\mathscr{C}^{\prime}$.

Proof. As in the definition of $F(\mathscr{C})$ in $\S I$, we pick a set $u_{i}, 1 \leqq i \leqq m+1, m=2^{k-1}$, where $2^{k}=\operatorname{dim} \mathscr{C}$, and denote by $\mathscr{B}$ the composition subalgebra of $\mathscr{C}$ spanned by $u_{i}$, $1 \leqq i \leqq m$. We may assume, by Lemma 1 , that $u_{1}=1$ and hence

$$
\lambda(u)=N\left(\sum_{2}^{m} x_{i} u_{i}+u_{m+1}\right) .
$$

Proof of 1 . If $\mathscr{C}$ is split, Lemma 3 yields the result since $F(\mathscr{C})=L(\mathscr{C})$ is a rational function field in $m-1$ indeterminates over $K$. If $\mathscr{C}$ is not split, neither is $\mathscr{C}_{L(\mathscr{C})}$ by Lemma 3 , and since $\lambda(u)$ is by definition $N\left(\sum_{2}^{m} x_{i} u_{i}+u_{m+1}\right)$, where $\sum_{2}^{m} x_{i} u_{i}+u_{m+1}$ $\in(L(\mathscr{C}) 1)^{\perp}$, Lemma 5 yields the result. 
Proof of 2. If there is a $K$ place from $F(\mathscr{C})$ to $F \cup \infty, \mathscr{C}_{F}$ is split, by Lemma 4 and Part 1 of this theorem.

If $\mathscr{C}$ is split, $\mathscr{C}_{F}$ is split for any $F \supseteq K$. By Lemma 2, there is a $K$-place of $F(\mathscr{C})=K\left(x_{2}, \ldots, x_{m}\right)$ into $F \cup \infty$ for any $F \supseteq K$ as desired.

Suppose $\mathscr{C}$ is not split, $\mathscr{C}_{F}$ is split. By (5), $\mathscr{C}_{F}$ contains a totally isotropic subspace $W$ of dimension $m$ over $F$. By a dimensionality argument $W$ intersects $F u_{1}+\cdots$ $+F u_{m+1}$ so there is $u=\beta u_{1}+\sum_{2}^{m+1} \beta_{i} u_{i}$ in $\mathscr{C}_{F}, u \neq 0$, with $N_{F}(u)=0$. Thus

$$
\beta^{2}=-\sum_{2}^{m+1} \beta_{i}^{2} N\left(u_{i}\right)
$$

and, if $\beta_{m+1} \neq 0$,

$$
\left(\beta \beta_{m+1}^{-1}\right)^{2}=-\sum_{2}^{m}\left(\beta_{i} \beta_{m+1}^{-1}\right)^{2} N\left(u_{i}\right)-N\left(u_{m+1}\right) .
$$

By Lemma 2, corollary, there is a $K$-place $\varphi$ of $F(\mathscr{C})=K\left(x_{2}, \ldots, x_{m}\right)\left((-\lambda(u))^{1 / 2}\right)$ into $F \cup \infty$ mapping $x_{i}$ to $\beta_{i} \beta_{m+1}^{-1},(-\lambda(u))^{1 / 2}$ to $\pm \beta \beta_{m+1}^{-1}$.

If $\beta_{m+1}=0$, some $\beta_{i}, i \neq m+1$ must be nonzero, since $0=\beta^{2}+\sum_{2}^{m} \beta_{i}^{2} N\left(u_{i}\right)$ and not all of $\beta, \beta_{i}$ are zero. We assume, without loss of generality, that $\beta_{m} \neq 0$. Then $\left(\beta \beta_{m}^{-1}\right)^{2}=\sum_{2}^{m}\left(\beta_{i} \beta_{m}^{-1}\right)^{2} N\left(u_{i}\right)$. Again by the corollary to Lemma 2 , there is a $K$-place of $F(\mathscr{C})=K\left(x_{2}, \ldots, x_{m}\right)\left((-\lambda(u))^{1 / 2}\right)=K\left(x_{2} x_{m}^{-1}, \ldots, x_{m-1} x_{m}^{-1}, x_{m}^{-1}\right)\left(x_{m}^{-1}(-\lambda(u))^{1 / 2}\right)$ into $F \cup \infty$ mapping $x_{i} x_{m}^{-1}$ to $\beta_{i} \beta_{m}^{-1}, 2 \leqq i<m, x_{m}^{-1}$ to zero, and $\left(x_{m}^{-1}(-\lambda(u))^{1 / 2}\right)$ to $\pm \beta \beta_{m}^{-1}$, since $x_{i} x_{m}^{-1}, 2 \leqq i<m, x_{m}^{-1}$ are algebraically independent over $K$.

Proof of 3 . If $\mathscr{C}$ is split, $F(\mathscr{C})$ is a rational function field over $K$ and hence, if $\mathscr{C}_{F(\mathscr{C})}^{\prime}$ is split, $\mathscr{C}^{\prime}$ is split over $K$ by Lemma 3.

If $\mathscr{C}, \mathscr{C}^{\prime}$ are not split over $K$ and $\mathscr{C}_{F(\mathscr{C})}^{\prime}$ is split, then since $\mathscr{C}_{L(\mathscr{C})}$ is not split and $F(\mathscr{C})$ is a quadratic extension of $L(\mathscr{C})$, Lemma 5 implies there is $u^{\prime} \in\left(1^{\prime}\right)^{\perp} \subseteq \mathscr{C}_{L(\mathscr{C})}^{\prime}$ such that $N_{L(\mathscr{C})}^{\prime}\left(u^{\prime}\right)=\lambda(u)$. Thus in $\mathscr{C}_{L(\mathscr{C})\left(x_{1}\right)}^{\prime}$,

$$
N_{L(\mathscr{C})\left(x_{1}\right)}^{\prime}\left(x_{1} 1+u^{\prime}\right)=x_{i}^{2}+\sum_{2}^{m} x_{i}^{2} N\left(u_{i}\right)+N\left(u_{m+1}\right) .
$$

It follows easily from a result of Pfister ([5], Satz 3) that the subspace $K u_{1}+\cdots$ $+K u_{m+1}$ is equivalent to a subspace of $\mathscr{C}^{\prime}$. By the Corollary to Lemma $6, \mathscr{C}$ is isomorphic to a subalgebra of $\mathscr{C}^{\prime}$.

We note finally that, in the event $\operatorname{dim} \mathscr{C}=4$, i.e., when $\mathscr{C}$ is a generalized quaternion algebra over $K$, a judicious choice of the elements $u_{i}$ in the definition of $F(\mathscr{C})$ will give rise to the same splitting field obtained by Witt [7].

\section{BIBLIOGRAPHY}

1. A. A. Albert, Quadratic forms permitting composition, Ann. of Math. 43 (1942), 161-177.

2. S. A. Amitsur, Generic splitting fields of central simple algebras, Ann. of Math. 62 (1955), 8-43.

3. M. Deuring, Lectures on the theory of algebraic functions of one variable, Tata Inst. of Fund. Res., Bombay, 1959. 
4. N. Jacobson, Composition algebras and their automorphisms, Rend. Circ. Mat. Palermo (2) 7 (1958), 55-80.

5. A. Pfister, Multiplikative quadratische formen, Arch. Math. 16 (1965), 363-371.

6. P. Roquette, On the Galois cohomology of the projective linear group and its applications to the construction of generic splitting fields of algebras, Math. Ann. 150 (1963), 411-439.

7. E. Witt, Uber ein Gegenbeispiel zum Normensatz, Math. Z. 39 (1934-1935), 462-567.

The Oho State University, Columbus, Оhо 